\title{
SOME ASYMPTOTIC PROPERTIES OF BONUS SYSTEMS
}

\author{
K. LOIMARANTA \\ Helsinki
}

\section{Introduction}

In motorcar insurance-at least in Europe-is widely used a merit rating system called bonus (or malus) system, characterized among others by the fact that only the number of claims occurred does modify the premium. A great variety of different bonus systems is in use. Each country-if not each company-seems to prefer a different bonus system and there is a lack of agreement whether one of them is better than the other. Efforts to construct a theoretically optimal bonus system have not been able to change the situation. General methods to evaluate and compare different existing or proposed bonus systems are therefore wanted.

In the present paper the theory of Markow chains is used to develope formulas for some asymptotic properties of bonus systems. Quantities: efficiency of a bonus system, discrimination power of bonus rules and minimum variance bonus scale are introduced. The last of them gives an asymptotical solution for the problem to find locally "best" bonus scales for given bonus rules. Finally the Danish bonus system is used as an illustration.

\section{Bonus system as Markov chain}

We call a merit rating system bonus system if the following assumptions are valid:

i. All policies of a given risk group can be devided into a finite number of classes so that the premium of a policy for a given period depends solely on the class for that period.

ii. The actual class is uniquely defined by the class for the previous period and the number of claims occurred (regulated) during the period.

iii. There exists a last class where all policies will be placed after a number of claimfree periods great enough. 
To specify a bonus system it is thus necessary to know two factors:

i. Bonus rules, i.e., the rules which give the new class when the old class and the number of claims for the period are known. The rules define also the number of classes. These rules can be given in the form of transformations $T_{k}$ defined so that $T_{k}(i)=j$, if the policy will be moved from class $i$ to class $j$ after $k$ claims.

ii. Bonus scale, which means the premiums $b_{i}$ for all bonus classes $i$. We assume that these premiums are given as a vector $B$ with components $b_{i}$.

We presume that premiums considered in this paper are premiums net of charges if not contrary asserted. Passage on premiums gross of charges is mostly trivial.

The transformation $T_{k}$ can be written as a matrix

$$
T_{k}=\left(t_{i j}^{(k)}\right) \text {, }
$$

where

$$
t_{i j}^{(k \prime}=\mathrm{I}, \quad \text { if } \quad T_{k}(i)=j
$$

otherwise

$$
t_{i j}^{(k)}=0 \text {. We have obviously }
$$

$$
\begin{gathered}
t_{i j}^{(k)} \geqslant 0 \\
\sum t_{i j}^{(k)}=\mathrm{I} \\
, \quad t^{\prime}
\end{gathered}
$$

We assume now that the claim frequency, i.e., the expected number of claims per period for a given policy, is $\lambda$ and that the probability distribution of the number of claims during one period, $p_{k}(\lambda)$ is uniquely defined by the parameter $\lambda$. We assume further that the value of $\lambda$ is independent of time.

The probability that a policy placed in class $i$ will be moved to class $j$ for the next period is thus

$$
p_{i j}(\lambda)=\sum_{k} p_{k}(\lambda) t_{i j}^{(k)}
$$

From eq. (2) it follows immediately

$$
\left\{\begin{aligned}
p_{i j}(\lambda) & \geqslant 0 \\
\Sigma p_{i j}(\lambda) & =\mathrm{I}
\end{aligned}\right.
$$


The matrix

$$
M(\lambda)=\left(p_{i j}(\lambda)\right)=\sum_{k} p_{k}(\lambda) T_{k}
$$

is then the transition matrix of a Markov chain.

From the assumption iii. on page $I$ we have, if $s$ is the last class,

$$
T_{k}^{n}(i)=s
$$

for $n \geqslant n_{i}$. Because the number of classes is finite, eq. (6) is valid for $n \geqslant n_{0}$, where $n_{0}=\operatorname{Max}\left(n_{i}\right)$. If now the probability for zero claims is positive, $p_{0}(\lambda)>0$, the probability to move from class $i$ to class $s$ during $n_{0}$ periods is

$$
p_{i j}^{\left(n_{0}\right)} \geqslant p_{0}^{n_{0}}>0
$$

This is, according to the theory of Markov chains, sufficient condition for the "regular case". We have thus the following result:

If the probability distribution for the number of claims for a given policy during one period is independent of the period, the successive bonus classes for that policy form a Markov chain with transition matrix $M(\lambda)$ given by eq. (5).

If the probability for a claimfree period is positive $p_{0}(\lambda)>0$ we have the regular case.

In the continuation the probability distribution $p_{k}(\lambda)$ is assumed to satisfy these requirements so that we have a regular case of a Markov chain to consider.

\section{Mean premium and excess premiums}

We start with some well-known results from the theory of Markov chains.

In the regular case the value one is a simple eigenvalue of the matrix $M$. The corresponding left hand eigenvector, row vector $A=\left(a_{i}\right)$, is uniquely defined by equations

$$
\left\{\begin{aligned}
A & =A M \\
\Sigma a_{i} & =\mathrm{I}
\end{aligned}\right.
$$

The quantities $a_{i}$ are the asymptotic probabilities for the different classes, i.e., $a_{i}$ is the limit value for the probability for the policy to be in class $i$, when the number of periods $n \rightarrow \infty$. 
Let $x_{i}^{(n)}$ be the premium a policy holder has to pay during $n$ periods when he starts from class $i$. As depending on the future claims it is a stochastic variable. The theory of Markov chains gives the mean value and the mean square deviation for it.

$$
\left\{\begin{array}{l}
E\left(x_{i}^{(n)}\right)=b . n+g_{i}+\varepsilon_{i, n} \\
\sigma^{2}\left(x_{i}^{(n)}\right)=\sigma^{2} n+c_{i}+\varepsilon_{i, n}
\end{array}\right.
$$

where $\varepsilon_{i, n}$ and $\varepsilon_{i, n}$ converge exponentially to zero with $n$. The distribution function of $x_{(n)}^{i}$ is asymptotically normal if $\sigma^{2} \neq 0$.

We can see from eq. (8) that the limit value for the premium per period is $b$, called mean premium. It is independent on the initial class $i$ and can be calculated from equation

$$
b=\Sigma_{i} a_{i} b_{i}=A B .
$$

The remaining part of the first eq. (8), $g_{\ell}+\varepsilon_{\ell, n}$, is the expected value of the extra premium the policy holder has to pay because he starts from class $i$. Its limit value $g_{i}$ is called excess premium in that class.

For the excess premiums we have the recurrence equations

$$
g_{i}=b_{i}-b+\sum_{j} p_{i j} g_{j}
$$

or if $G$ is a vector with components $g_{i}$ we have

$$
\left\{\begin{array}{l}
G=B-b J+M G \\
\sum a_{i} g_{j}=0
\end{array}\right.
$$

where $J$ is a vector with all components $=\mathrm{I}$. The last equation is needed because the other equations are linearly dependent.

From the theory of Markov chains we know further the expression for the limit value of the mean square deviation

$$
\sigma^{2}=\sigma_{0}^{2}+2 \sum_{i} a_{i} b_{i}\left(g_{i}-b_{i}+b\right)
$$

where

$$
\sigma_{0}^{2}=\sum_{i} a_{i}\left(b_{i}-b\right)^{2}
$$

is the mean square deviation for one period premium. 
The mean premium, excess premiums and mean square deviations are naturally dependent on the claim frequency $\lambda$.

\section{Efficiency of a bonus system}

According to common conception the main purpose of a bonus system is to decrease the premiums for good risks and increase them for bad risks. It is thus a primary interest to know how well a bonus system works in this respect.

A measure for the risk is the risk premium $R=\lambda V$, where $V$ is the mean claim amount. $V$ is by experience practically constant inside one risk group and the risk premium depends thus solely on the claim frequency $\lambda$. In a reasonable bonus system the mean premium $b(\lambda)$ is an increasing function of $\lambda$. In an ideal case it equals the risk premium and $b(\lambda)$ is thus proportional to $\lambda$. A fractional change in claim frequency $d \lambda / \lambda$ causes in the mean premium a fractional change $d b / b$. In the ideal case these two changes equal. In general this is not true, the change $d b / b$ is smaller and the bonus system is thus less efficient. Consequently we can define the efficiency of a bonus system as the quotient of these two fractional changes, or

$$
\eta=\frac{\lambda}{b} \frac{d b}{d \lambda}=\frac{d \log b}{d \log \lambda}
$$

Thus for a reasonable bonus system is $\eta \geqslant 0$ and for an ideal system $\eta=I$. In general $\eta$ is between the values zero and one. Theoretically $\eta$ can of course be $>I$, but in practice such overefficient systems seem to be rare.

To calculate $\eta$ we need the value of

$$
\frac{d b}{d \lambda}=\sum_{i} \frac{d a_{i}}{d \lambda} b_{i} .
$$

The equations defining the derivatives $d a_{i} / d \lambda$ are attained by derivating eq. $(7)$

$$
\left\{\begin{array}{l}
\frac{d A}{d \lambda}=\frac{d A}{d \lambda} M+A \frac{d M}{d \lambda} \\
\sum_{i} \frac{d a_{i}}{d \lambda}=0
\end{array}\right.
$$


The matrix $d M / d \lambda$ is easily calculated for generally used probability distributions. E.g. in the case of Poisson distribution we have

$$
\frac{d M(\lambda)}{d \lambda}=\sum_{k=0}^{\infty} e^{-\lambda} \frac{\lambda^{k}}{k !}\left(T_{k+1}-T_{k}\right)
$$

Solution of the linear equation system (I4) is then not difficult.

For great values of $\lambda$ the quantity $\log b(\lambda)$ approaches-if it is monotone-to a definite limit because $b(\lambda)$ is bounded by $\operatorname{Max}\left(b_{j}\right)$. The quantity $\eta$, the derivative of $\log b(\lambda)$, goes thus to zero when $\lambda \rightarrow \infty$. If $b(0)>0$, we see that $\eta$ approaches to zero also when $\lambda \rightarrow 0$.

We have thus the following result:

The efficiency $\eta$ of a bonus system is in general positive for all values of $\lambda$ in the interval $(0, \infty)$. It goes nevertheless to zero when $\lambda \rightarrow \infty$ and also for $\lambda=0$, unless $b(0)=0$. We can thus in general obtain a good efficiency only for an interval $\lambda_{1} \leqslant$ $\lambda \leqslant \lambda_{2}$ where $\lambda_{1}>0$ and $\lambda_{2}<\infty$.

Comment: The case $b(0)=0$ is perhaps not quite unrealistic because $b$ is the premium net of charges. The gross premium also for $b=0$ will be positive.

The behaviour of the premium $b(\lambda)$ for moderate values of $\lambda$ is of course more important than the limits of the efficiency for $\lambda=0$ and $\infty$.

We consider now the equation

$$
b(\lambda)=\lambda V .
$$

It states that the net premium equals the risk premium. When $\lambda$ increases from zero to infinity, the left hand side grows up from some positive value $b(0)$ toward a finite limit $b(\infty)$, as we have assumed. The eq. (I6) has thus at least one solution $\lambda_{0}$, for which $b\left(\lambda_{0}\right)=\lambda_{0} V$. 
Using this value $\lambda_{0}$ as the initial value we can integrate eq. (I3) and get the premium $b(\lambda)$ as a function of $\lambda$

$$
\left\{\begin{array}{l}
b(\lambda)=\lambda V e^{\int^{\lambda_{0}}(1-n(\lambda)) d \log \lambda} ; \lambda \leqslant \lambda_{0} \\
b(\lambda)=\lambda V e^{\int_{\lambda_{0}}^{\lambda}(1-n(\lambda)) d \log \lambda} ; \lambda \geqslant \lambda_{0}
\end{array}\right.
$$

If $\eta(\lambda)<I$, the integrales in exponents are positive. Then $b(\lambda)>\lambda V$ for $\lambda<\lambda_{0}$ and $b(\lambda)<\lambda V$ for $\lambda>\lambda_{0}$. The solution $\lambda_{0}$ of the eq. (I6) is thus unique. We can call it the central value of $\lambda$ for that risk group. We sum up the results:

If the efficiency $\eta(\lambda)$ of a bonus system is less than one for all values of $\lambda$, there exists one and only one value of $\lambda$, called central value, $\lambda_{0}$, for which net premium and risk premium equal. For values $\lambda<\lambda_{0}\left(\lambda>\lambda_{0}\right)$ the net premium is greater (less) than the risk premium.

\section{Discrimination power and minimum variance bonus scale}

Calculation of the efficiency of different bonus systems in use exhibits that it is for common values of $\lambda$ remarkably low. Nevertheless it is not at all difficult to construct bonus systems with efficiency one, simply by keeping the bonus rules unchanged and making the bonus scale steep enough. The primary disadvantage of such system seems to be that the random variation of the premium from period to period will be very high and the whole idea of insurance, to give economical security, is to great extent lost. It is thus natural by the choice of a bonus scale to search the lowest value for the mean square deviation of one period premium when mean premium and efficiency are given. Because known values for claim frequency, mean premium and efficiency according to eq. (I3) imply also a known value of the derivative $d b / d \lambda$, we have the following problem:

Find the minimum for

$$
\sigma_{0}^{2}=\sum_{i} a_{i}\left(b_{i}-b\right)^{2}
$$


when

$$
\begin{gathered}
\sum_{i} a_{i} b_{i}=b \\
\sum_{i} \frac{d a_{i}}{d \lambda} b_{i}=\frac{d b}{d \lambda}=\text { const. }
\end{gathered}
$$

Using the Lagrange method the derivatives of the function

$$
F=\sum_{i} a_{i}\left(b_{i}-b\right)^{2}-2 c_{1}\left(\sum_{i} a_{i} b_{i}-b\right)-2 c_{2}\left(\sum_{i} \frac{d a_{i}}{d \lambda} b_{i}-\frac{d b}{d \lambda}\right)
$$

must be zero

$$
\frac{\mathrm{I}}{2} \frac{\partial F}{\partial b_{i}}=a_{i}\left(b_{i}-b\right)-c_{1} a_{i}-c_{2} \frac{d a_{i}}{d \lambda}=0 .
$$

The value of the constant $c_{1}$ is easily determined

$$
\sum_{i} \frac{I}{2} \frac{\partial F}{\partial b_{i}}=-c_{1}=0
$$

The solution is thus ${ }^{1}$ )

$$
b_{i}=b+c_{2} \frac{\mathrm{I}}{a_{i}} \frac{d a_{i}}{d \lambda} .
$$

We introduce notations

$$
\beta_{i}=\frac{\lambda}{a_{i}} \frac{d a_{i}}{d \lambda}
$$

and can see that each linear transformation of the quantities $\beta_{i}$

$$
b_{i}=b+c \beta_{i}
$$

is a solution of the minimizing problem for some values of the conditioning quantities $b$ and $d b / d \lambda$. A bonus scale corresponding to premiums (I9) will be called minimum variance bonus scale.

The mean premium for the solution (Ig) is of course $b$ and the derivative will be

$$
\frac{d b}{d \lambda} \sum_{i} \frac{d a_{i}}{d \lambda} b_{i}=c \lambda \sum_{i} \frac{\mathrm{I}}{a_{i}}\left(\frac{d a_{i}}{d \lambda}\right)^{2}
$$

1) If some $a_{1}=0$ the value of the corresponding premium $b_{i}$ is irrelevant. 
Also the mean square deviation is easily calculated

$$
\sigma_{0}^{2}=\sum_{i} a_{i}\left(b_{i}-b\right)^{2}=c^{2} \lambda^{2} \sum_{i} \frac{I}{a_{i}}\left(\frac{d a_{i}}{d \lambda}\right)^{2} .
$$

Using the notation

$$
d^{2}=\sum_{i} a_{i} \beta_{i}^{2}=\lambda^{2} \sum_{i} \frac{I}{a_{i}}\left(\frac{d a_{i}}{d \lambda}\right)^{2}
$$

we have thus for the premiums (rg)

$$
\begin{aligned}
\frac{d b}{d \lambda} & =\frac{c}{\lambda} d^{2} \\
\sigma_{0}^{2} & =c^{2} d^{2} \\
\eta & =c \frac{d^{2}}{b}=d \frac{\sigma_{0}}{b}
\end{aligned}
$$

We see that the efficiency of these bonus systems is a product of two factors, the relative variance $\sigma_{0} / b$ and a factor $d$ which does not depend on the actual bonus scale. The quantity $d^{2}$ will be called the discrimination power of the bonus rules.

Let us now consider an arbitrary bonus scale. We split up the corresponding $b$-vector into two components

$$
b_{i}=b+c \beta_{i}+h_{i}
$$

so that the first component $b+c \beta_{i}$ is of the form discussed before The second component $h_{i}$ with mean value zero has to be orthogonal to the $\beta$-vector. This is achieved by giving for $c$ the value

$$
c=\frac{\mathrm{I}}{d^{2}} \sum_{i} a_{i} \beta_{i} b_{i}=\frac{\lambda}{d^{2}} \frac{d b}{d \lambda}=\frac{\eta b}{d^{2}} .
$$

The mean square deviation can now be splitted up in corresponding way

$$
\sigma_{0}^{2}=\sum_{i} a_{i}\left(c \beta_{i}+h_{i}\right)^{2}=\left(\frac{\eta b}{d}\right)^{2}+\sum_{i} a_{i} h_{i}^{2}
$$

This gives the value of

$$
\eta^{2}=\frac{d^{2}}{b^{2}}\left(\sigma_{0}^{2}-\sum_{i} a_{i} h_{i}^{2}\right) .
$$


We have hence the following result:

The efficiency of a bonus system satisfies the inequality

$$
\eta \leqslant a \frac{\sigma_{0}}{b}
$$

where the discrimination power of the bonus rules, $d$ given by eq. (20) is independent of the bonus scale. The sign of equality holds if and only if the premiums for different bonus classes form a minimum variance bonus scale, i.e., if they are a linear transformation of the scale $\left(\beta_{i}\right)$ defined by eq. (I 8 ).

Comment. We have so far discussed only the premiums net of charges. The charges are generally divided to a constant part $\gamma_{1}$ and to a proportional part $\gamma_{2}$ so that the gross premiums are

$$
b_{i}=\gamma_{1}+\left(\mathrm{I}+\gamma_{2}\right) b_{i} .
$$

Because there is a linear relation between the net and gross premiums, both of them give coincidentally a minimum variance bonus scale. The proposition above is thus valid also for gross premiums if we replace the inequality (23) by

$$
\eta \leqslant d \frac{\sigma_{0}^{\prime}}{b^{\prime}-\gamma_{1}}
$$

where $\sigma_{0}^{\prime}$ is the mean square deviation of gross premiums.

\section{A numerical example}

Let us consider a concrete example, e.g. the Danish bonus system.

It has four bonus classes labeled o to 3 . The premiums for them decrease in a geometrical serie with the ratio $3 / 4$.

For every claimfree year the policy advances one class up to the last bonus class, number 3 . After one claim the policy will be moved from classes 2 and 3 to class I. After two (or more) claims during one year or one claim during each of two successive years the new class is 0 . New policies will be placed in the class one.

We see that the new bonus class might depend directly on two years old claims and the assumption $i i$. in our definition of a bonus system is violated. To put the things in order we add to the class label a second digit, which is o or I depending on whether there was 
a claim in the year before or not. The possible bonus classes are then oo, Io, II, 2I and 3I. All requirements to be a bonus system as we have defined it are now performed.

The transformations $T_{k}$ for bonus rules and the vector $B$ defining the relative premiums are

\begin{tabular}{lllcl}
\hline class & $T_{0}$ & $T_{1}$ & $T_{k} ; k \geqslant 2$ & $B$ \\
\hline oo & I I & oo & oo & $4 / 3$ \\
Io & 2I & oo & oo & I \\
I I & 2I & 10 & oo & I \\
2 I & 3I & 10 & oo & $3 / 4$ \\
3I & 3I & Io & oo & $9 / 16$ \\
\hline
\end{tabular}

We assume that the number of claims follows Poisson distribution

$$
p_{k}(\lambda)=e^{-\lambda} \frac{\lambda^{k}}{k !}
$$

The matrix $M(\lambda)$, eq. (5) is then

$$
M(\lambda)=\left\{\begin{array}{lllll}
\mathrm{I}-e^{-\lambda} & 0 & e^{-\lambda} & 0 & 0 \\
\mathrm{I}-e^{-\lambda} & 0 & 0 & e^{-\lambda} & 0 \\
\mathrm{I}-(\mathrm{I}+\lambda) e^{-\lambda} & \lambda e^{-\lambda} & 0 & e^{-\lambda} & 0 \\
\mathrm{I}-(\mathrm{I}+\lambda) e^{-\lambda} & \lambda e^{-\lambda} & 0 & 0 & e^{-\lambda} \\
\mathrm{I}-(\mathrm{I}+\lambda) e^{-\lambda} \lambda e^{-\lambda} & 0 & 0 & e^{-\lambda}
\end{array}\right\}
$$

The equation systems for limit probabilities eq. (7) and for excess premiums eq. (IO) can in this case be solved in closed form, but also a direct numerical solution for a given value of $\lambda$ is easily calculated. E.g. $\lambda=0.2$ gives us

\begin{tabular}{ccc}
\hline class & \multicolumn{1}{c}{$a_{i}$} & \multicolumn{1}{c}{$g_{i}$} \\
\hline oO & $0.047^{2} \mathbf{I}$ & $\mathbf{1 . 1 5 5 ^ { 8 } 7}$ \\
IO & 0.13406 & $0.492 \mathrm{I} 7$ \\
II & 0.03865 & 0.38349 \\
2I & $0.144^{\circ}$ & -0.02002 \\
3I & 0.63868 & -0.20752 \\
\hline$b=$ & 0.70096 & \\
$\sigma_{0}{ }^{2}=$ & 0.046907 &
\end{tabular}

The excess premium for new policies (class II) is thus $54.7 \%$ of the mean premium, a relatively low value. 
To calculate the efficiency from eq. (I3) and eq. (I4) we need the matrix $d M / d \lambda$. It can be found either by derivation from the matrix $M(\lambda)$ above or from eq. (15).

$$
\frac{d M(\lambda)}{d \lambda}=\left\{\begin{array}{cclll}
e^{-\lambda} & 0 & -e^{-\lambda} & 0 & 0 \\
e^{-\lambda} & 0 & 0 & -e^{-\lambda} & 0 \\
\lambda e^{-\lambda} & (\mathrm{I}-\lambda) e^{-\lambda} & 0 & -e^{-\lambda} & 0 \\
\lambda e^{-\lambda} & (\mathrm{I}-\lambda) e^{-\lambda} & 0 & 0 & -e^{-\lambda} \\
\lambda e^{-\lambda} & (\mathrm{I}-\lambda) e^{-\lambda} & 0 & 0 & -e^{-\lambda}
\end{array}\right\}
$$

When the derivatives $d a_{i} / d \lambda$ are known the calculation of the minimum variance bonus scale is an easy task.

For the former value $\lambda=0.2$, we get

\begin{tabular}{rrr}
\hline class & $d a_{i} / d \lambda$ & \multicolumn{1}{c}{$\beta_{i}$} \\
\hline oo & $0.4 \mathbf{I} 654$ & $\mathbf{I} .76476$ \\
Io & 0.40219 & 0.60000 \\
I I & 0.30238 & $\mathbf{I} .56476$ \\
$2 \mathbf{I}$ & 0.43545 & 0.61589 \\
$3 \mathbf{I}$ & $-\mathbf{I} .55656$ & -0.48744 \\
\hline$d b / d \lambda=$ & 0.71098 & \\
$\eta=$ & 0.20286 &
\end{tabular}

The efficiency $20 \%$ for $\lambda=0.2$ is very low, but not much worse than usually.

Also the value of discrimination power, eq. (20) might be of interest

$$
d^{2}=0.49529 .
$$

The actual bonus scale $\left(b_{i}\right)$ can now be compared with the minimum variance bonus scale $\left(b+c \beta_{i}\right)$, where $c=\eta b / d^{2}=0.287$ Io.

\begin{tabular}{ccc}
\hline class & $b_{i}$ & $b+c \beta_{i}$ \\
\hline oo & 1.33333 & $\mathbf{1} .20763$ \\
Io & $\mathrm{I} .00000$ & 0.87322 \\
I I & $\mathrm{I} .00000$ & $1.1502 \mathrm{I}$ \\
$2 \mathrm{I}$ & 0.75000 & 0.87779 \\
$3 \mathrm{I}$ & 0.56250 & 0.56102 \\
\hline
\end{tabular}


Both of these bonus scales have the same mean premium $b=$ 070096 and the same efficiency $\eta=0.20286$, but the variancies are 0046907 resp 0040825 The minimum variance bonus scale has thus I $3 \%$ lower variance than the used bonus scale The discrepance between the premiums for classes Io and II in the minimum variance bonus scale is remarkable

\section{BIBLIOGRAPHIE}

[r] BichseL, F, Erfahrungs - Tariffierung in der Motorfahrzeug haftpflicht - Versicherung Mitt Verein schweiz Versicherungsmath 64, I964

[2] Buhlmans, H, Optımale Pramienstutensysteme Mitt Vereın schweız Versicherungsmath 64,1964

[3] Delaporte, P, Quelques problèmes de statistıques mathématıques posés par l'Assurance Automobile et le Bonus pour non sinistre Bull Trım Inst Actuaires Franca1s 70 , 1959

Tarification du risque individuel d'accidents d'automobiles par la prime modelée sur le risque, ASTIN Bull III 3, 1965

[4] Depoin, P, Étude de la Fréquence et de la Bonification pour non sinistre dans un portefeulle ,Tous risques modernes” Bull Trım Inst Actualres Francals 70, 1959

Applications de la statistique aux assurances accidents et dommages Paris 1967

[5] Decron, M, A Theoretical Study of the No-Claim Bonus Problem ASTIN Bull III I, 1963

[6] Dubois de Montregnaud, B, La prime ajustable pour la tarification de la responsabilité cıvile automobile Bull Trım Inst Actuarres Francais 77,1966

[7] Franckx, E, Théorie du bonus ASTIN Bull I 3, 1960

[8] FrÉCHeT, M, Méthode des fonétions arbitraires Théonie des événements en chaine dans le cas d'un nombre finı d'états possibles Parıs 1938

Essa1 d'une étude des successions de sinistres considérés comme processus stochastıque Bull Trum Inst Actuaires Francais 70, 1959

[9] Grenander, U, Some Remarks on Bonus Systems in Automobile Insurance Skand Aktuarietıdskrift, I957

[10] Gurtler, ML, Bonus ou malus ? ASTIN Bull III I, 1963

[II] LundBerG, $O$, Une note sur des systèmes de tarification basés sur des modèles du type Poisson composé ASTIN Bull IV I, I966

[i2] Molnar, D and Rockwell, T, Analysis of Policy Movement in a Merit Rating Program an Application of Markov Processes J Risk Ins 33,1966

[13] Pesonen, E, A Numerical Method of Finding a Suitable Bonus Scale ASTIN Bull II I, I 962

[14] SEAL, M, Stochastic Theory of a Risk Business New York 1969

[15] Contribution a l'etude du bonus pour non sinistre en assurance automobile ASTIN Bull I 3, 1960 